1 Hacettepe Journal of Mathematics and Statistics

$\bigcap$ Volume 47 (6) (2018), 1652-1670

\title{
Poisson-odd generalized exponential family of distributions: theory and applications
}

\author{
Mustapha Muhammad*
}

\begin{abstract}
In this paper, we introduce a new family of distributions called the Poisson-odd generalized exponential distribution (POGE). Various properties of the new model are derived and studied. The new distribution has the odd generalized exponential as its limiting distribution. We present and study two special cases of the POGE family of distributions, namely the Poisson odd generalized exponential-half logistic and the Poisson odd generalized exponential-uniform distributions. Estimation and inference procedure for the parameters of the new distribution are discussed by the method of maximum likelihood; we also evaluate the proposed estimation method by simulation studies. Applications to two real data sets are provided in order to demonstrate the performance of the proposed family of distributions.
\end{abstract}

Keywords: Odd generalized exponential distribution, moments, maximum likelihood estimates.

Mathematics Subject Classification (2010): 62E05, 62F10

Received : 21.08.2016 Accepted : 19.12.2016 Doi : 10.15672/HJMS.2016.393

\section{Introduction}

Exponential distribution is commonly used in solving many practical problems, especially in life time data analysis. In probability modeling, numerous families of distributions have been proposed and studied via different directions based on the exponential distribution. For instance, some distributions generalized (or extended) the exponential distribution, these includes the Weibull (W) distribution, linear failure rate distribution (LFR), generalized exponential distribution (GE) by [14], generalized linear failure rate (GLFR) by [35], generalized linear exponential (GLE) by [20], exponentiated generalized linear exponential (EGLE) by [34], the Nadarajah and Haghighi's (NHE) exponentialtype by [29], among other.

*Department of Mathematical Sciences, Bayero University Kano, Nigeria, Email: mmmahmoud12@sci.just.edu.jo, mmustaphamuhd@yahoo.com 
In another approach, a new family of probability models with more flexibility is generated by combining the continuous and discrete probability distributions such as, the exponential geometric (EG) distribution introduced by [3], [16] proposed the exponential Poisson (EP), exponentiated exponential Poisson (EEP) by [33], complementary exponentiated exponential geometric (CEEG) by [19], exponentiated exponential binomial(GEB) by [7], generalize exponential power series (GEPS) by [21], binomial exponential-2 (BE2) by [6], Poisson exponential (PE) by [10], generalized Gompertz-power series (GGPS) by [40], Lindley-Poisson (LP) by [13], bivariate Weibull-power series by [30] and Linear failure rate-power series (LFRPS) by [22]. Others that follow the same approach include the Weibull power series(WPS), extended Weibull power series (EWPS), exponentiated Weibull-logarithmic (EWL), exponentiated Weibull Poisson (EWP), exponentiated Weibull geometric (EWG) and exponentiated Weibull power series (EWPS) distributions proposed and studied by [27, 37, 24, 23, 25] and [26] respectively. Moreover, in recent years, some new generators of distributions based on the exponential distribution such as the odd-generalized exponential family of distributions (OGE) and odd- exponential-G family of distributions (OEG) were proposed and analyzed by [39] and [9] respectively. In this article, we propose a new family of distributions called the Poisson odd generalized exponential distribution (POGE), by joining together the odd generalized exponential family of distributions and the Poisson distribution. The rest of the paper is structured as follows. In section 2 the new POGE family of distribution is obtained, several properties of this model are derived and studied such as the explicit algebraic expression of its ordinary moments, order statistics, Shannon entropy, Renyi entropy and reliability. In section 3 maximum likelihood estimation for the model parameters is discussed. Two real applications are provided in section 4 . Section 5 provides conclusions.

\section{The POGE family of distributions}

Let $Y_{1}, Y_{2}, \cdots, Y_{n}$ be a random sample of size $N$ from odd generalize exponential distribution with cumulative distribution function (cdf) given by $J(y, \alpha, \beta, \zeta)=\left(1-e^{-\alpha \frac{G(y)}{G(y)}}\right)^{\beta}$, $y>0, \alpha, \beta>0$, where $G(y)=G(y ; \zeta)$ is the baseline cdf, $\zeta$ a vector parameter and $\bar{G}(y)=1-G(y)$. Let $N$ be a zero-truncated Poisson random variable independent of vector $Y$ with probability mass function given by $P(N=n)=\lambda^{n}\left(\left(e^{\lambda}-1\right) n !\right)^{-1}, \lambda>$ $0, n \in \mathbb{N}$. Let $X=\min \{Y\}_{i=1}^{N}$, then, the conditional random variable $(X \mid N=n)$ has the cdf $F(X \mid N=n)=1-\left[1-\left(1-e^{-\alpha \frac{G(x)}{G(x)}}\right)^{\beta}\right]^{n}$. Hence the marginal cdf of $X$ can be obtained as

$$
F(x ; \xi)=\frac{1-e^{-\lambda\left(1-e^{-\alpha \frac{G(x)}{G(x)}}\right)^{\beta}}}{\left(1-e^{-\lambda}\right)} \quad x>0,
$$

where $\alpha, \beta, \lambda>0, \xi$ a vector parameter and $\xi=(\alpha, \beta, \lambda, \zeta)$. A random variable $X$ with cdf given by (2.1), is denoted by $X \sim \operatorname{POGE}(\xi)$, and it's probability density function (pdf) is given by

$$
f(x ; \xi)=\frac{\alpha \beta \lambda g(x)}{\bar{G}(x)^{2}\left(1-e^{-\lambda}\right)} e^{-\alpha \frac{G(x)}{G(x)}}\left(1-e^{-\alpha \frac{G(x)}{G(x)}}\right)^{\beta-1} e^{-\lambda\left(1-e^{-\alpha \frac{G(x)}{G(x)}}\right)^{\beta}} .
$$


The followings are some new and existing members of $P O G E(\xi)$ family of distributions.

(1) If $\beta=1$, we have Poisson-odd exponential family of distributions (POE)

(2) If $G=\frac{x}{1+x}$ we have exponentiated exponential Poisson (EEP) by [33]

(3) If $\beta=1$ and $G=\frac{x}{1+x}$ we have exponential Poisson (EP) by [16]

(4) If $\alpha=\theta^{c}$ and $G=\frac{x^{c}}{1+x^{c}}$ we have exponentiated Weibull Poisson (EWP) with parameters $c, \beta, \theta$ and $\lambda$ by [26]

(5) If $\alpha=\theta^{c}, \beta=1$ and $G=\frac{x^{c}}{1+x^{c}}$ we have Weibull Poisson (WP) with parameters $c, \theta$ and $\lambda$ by [27]

(6) If $G=1-e^{-a x}$, we have Poisson-generalize Gompertz (PGG) distribution

(7) If $\beta=1$ and $G=1-e^{-a x}$, we have Poisson-Gompertz (PG)

The limiting distribution of the $P O G E(\xi)$ given by $(2.1)$ when $\lambda \rightarrow 0^{+}$is, $\lim _{\lambda \rightarrow 0^{+}} F(x ; \xi)=$ $\left(1-e^{-\alpha \frac{G(x)}{G(x)}}\right)^{\beta}$, which is the cdf of $O G E(\alpha, \beta, \zeta)$.

2.1. Special cases of POGE distribution. In this subsection, we present two special cases of the POGE family of distribution namely the Poisson odd generalized exponentialHalf logistic (POGE-HL) distribution and Poisson odd generalized exponential-Uniform (POGE-U) distribution which can be very useful in solving various problems in practical applications in the fields of sciences and applied sciences.

2.1.1. The POGE-Half Logistic (POGE-HL) distribution. The Poisson odd generalize exponential-Half Logistic (POGE-HL) distribution is obtained by choosing the baseline cdf and pdf in (2.1) and (2.2) to be Half Logistic distribution defined by $G(x)=\frac{\left(1-e^{-x}\right)}{\left(1+e^{-x}\right)}$ and $g(x)=\frac{2 e^{-x}}{\left(1+e^{-x}\right)^{2}}$ respectively. For $x>0$ and parameters $\alpha, \beta, \lambda>0$, the cdf and pdf of the POGE-HL distribution are given by

$$
\begin{aligned}
& F(x ; \alpha, \beta, \lambda)=\frac{1-e^{-\lambda\left(1-e^{-\alpha\left(\frac{e^{x}-1}{2}\right)}\right)^{\beta}}}{\left(1-e^{-\lambda}\right)}, \\
& f(x ; \alpha, \beta, \lambda)=\frac{\alpha \beta \lambda e^{x-\alpha\left(\frac{e^{x}-1}{2}\right)}}{2\left(1-e^{-\lambda}\right)}\left(1-e^{-\alpha\left(\frac{e^{x}-1}{2}\right)}\right)^{\beta-1} e^{-\lambda\left(1-e^{-\alpha\left(\frac{e^{x}-1}{2}\right)}\right)^{\beta}},
\end{aligned}
$$

respectively, where its limiting distribution is the odd-generalized exponential half logistic distribution (OGE-HL) when $\lambda \rightarrow 0^{+}$. Also when the shape parameter $\beta=1$ we obtain a new family of Poisson odd exponential-Half Logistic (POE-HL) distribution.

2.1.2. The POGE-Uniform (POGE-U) distribution. The Poisson odd generalized exponential - Uniform (POGE-U) distribution is obtained by taking the baseline cdf and pdf in $(2.1)$ and $(2.2)$ to be a continuous Uniform $(0, b)$ distribution defined by $G(x ; \zeta)=\frac{x}{b}$ and $g(x ; \zeta)=\frac{1}{b}$ respectively, where $\zeta=b$ and $0<x<b$. The cdf and pdf of the (POGE-U) distribution are given by

$$
\begin{aligned}
& F(x ; \alpha, \beta, \lambda, b)=\frac{1-e^{-\lambda\left(1-e^{-\alpha\left(\frac{x}{(b-x)}\right)}\right)^{\beta}}}{\left(1-e^{-\lambda}\right)}, \\
& f(x ; \alpha, \beta, \lambda, b)=\frac{\alpha \beta \lambda b e^{-\alpha\left(\frac{x}{(b-x)}\right)}}{(b-x)^{2}\left(1-e^{-\lambda}\right)}\left(1-e^{-\alpha\left(\frac{x}{(b-x)}\right)}\right)^{\beta-1} e^{-\lambda\left(1-e^{-\alpha\left(\frac{x}{(b-x)}\right)}\right)^{\beta}},
\end{aligned}
$$

respectively, where its limiting distribution is the odd-generalized exponential uniform distribution (OGE-U) when $\lambda \rightarrow 0^{+}$. When the shape parameter $\beta=1$ we obtain a new 
class of Poisson odd exponential-Uniform (POE-U) distribution.

Figure 1 display the plots of the density functions of the POGE-HL and POGE-U distributions for some selected values of parameters.
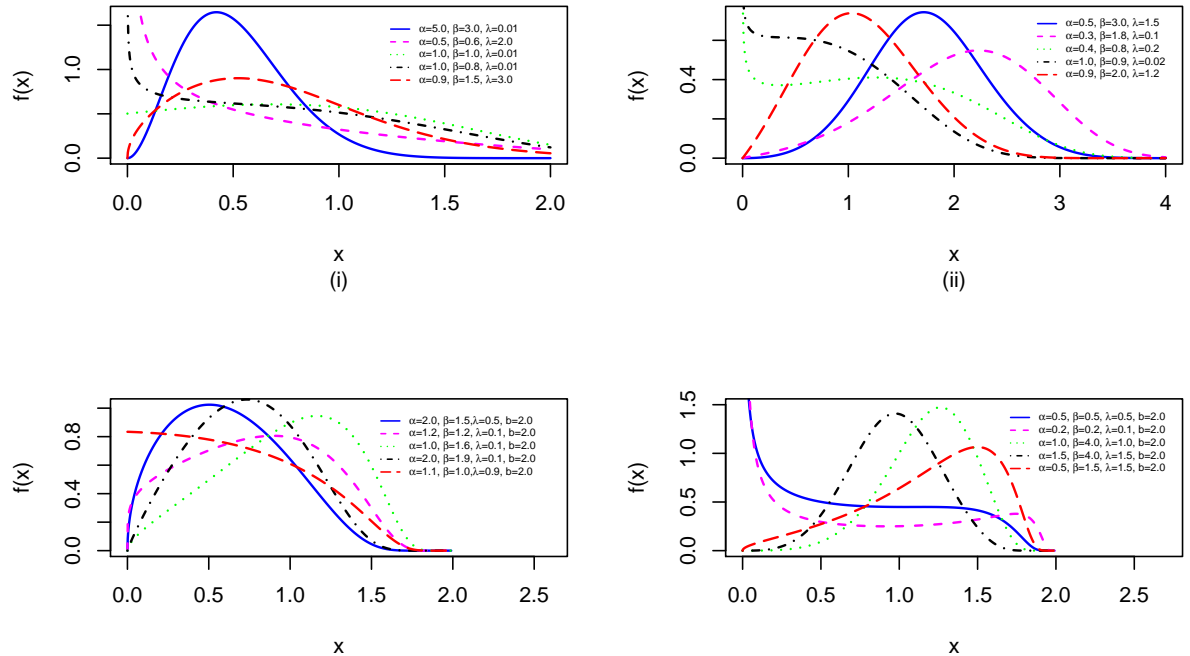

(iii)

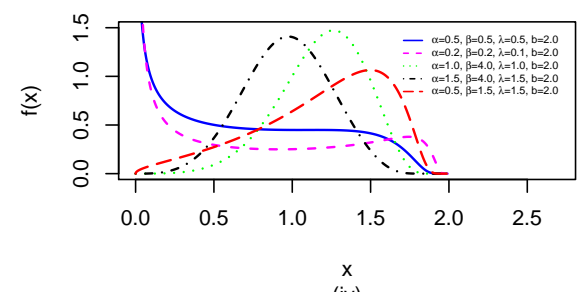

(iv)

Figure 1. pdf plots: POGE-HL (i) \& (ii) and POGE-U (iii) \& (iv).

2.2. Expansion of distribution. We provide a series representation of the POGE distribution based on certain conditions. First for $|z|<1$ and for $a>0$ real and non integer, we have

$$
(1-z)^{a-1}=\sum_{j=0}^{\infty} \frac{(-1)^{j} \Gamma(a)}{\Gamma(a-j) j !} z^{j}=\sum_{j=0}^{\infty}(-1)^{j}\left(\begin{array}{c}
a-1 \\
j
\end{array}\right) z^{j}
$$

By expanding the exponential expression $e^{-\lambda\left(1-e^{-\alpha \frac{G(x)}{G(x)}}\right)^{\beta}}$ in $(2.2)$, then, for $\beta(i+$ 1) $>0$ real and non integer we can apply (2.7), and after some algebraic manipulations, we obtain

$$
f(x)=\sum_{i=0}^{\infty} \sum_{j=0}^{\infty} \frac{(-1)^{i+j} \lambda^{i+1} \Gamma(\beta(i+1))}{\left(1-e^{-\lambda}\right) \Gamma(\beta(i+1)-j) i ! j !} \frac{\alpha g(x)}{\bar{G}(x)^{2}} e^{-\alpha(j+1) \frac{G(x)}{\bar{G}(x)}}
$$

Also by the exponential expansion of $e^{-\alpha(j+1) \frac{G(x)}{G(x)}}=\sum_{k=0}^{\infty} \frac{(-1)^{k} \alpha^{k}(j+1)^{k}}{k !} \frac{G(x)^{k}}{G(x)^{k}}$ in (2.8), then, applying generalized binomial expansion of $\bar{G}(x)^{-(k+2)}=(1-G(x))^{-(k+2)}=$ $\sum_{l=0}^{\infty}\left(\begin{array}{c}-(k+2) \\ l\end{array}\right)(-1)^{l} G(x)^{l}$, we get 


$$
\begin{aligned}
f(x) & =\sum_{i, j=0}^{\infty} \sum_{k, l=0}^{\infty}\left(\begin{array}{c}
\beta(i+1)-1 \\
j
\end{array}\right)\left(\begin{array}{c}
-(k+2) \\
l
\end{array}\right) \\
& \times \frac{(-1)^{i+j+k+l} \alpha^{k} \lambda^{i+1}(j+1)^{k}}{\left(1-e^{-\lambda}\right) i ! k !} g(x) G(x)^{k+l},
\end{aligned}
$$

thus,

$$
f(x ; \xi)=\sum_{i, j=0}^{\infty} \sum_{k, l=0}^{\infty} \varrho_{(i, j, k, l)} g^{*}(x ; k+l+1, \zeta),
$$

where,

$$
\varrho_{(i, j, k, l)}=\left(\begin{array}{c}
\beta(i+1)-1 \\
j
\end{array}\right)\left(\begin{array}{c}
-(k+2) \\
l
\end{array}\right) \frac{(-1)^{i+j+k+l} \alpha^{k} \lambda^{i+1}(j+1)^{k}}{\left(1-e^{-\lambda}\right)(k+l+1) i ! k !}
$$

and $g^{*}(x ; k+l+1, \zeta)$ is the density of the exponentiated $G(x ; \zeta)$ to the power of $k+l+1$

2.3. Quantile and moments. The $P^{t h}$ quantile of the $P O G E(\xi)$ distribution can be used for generating random data that follow the POGE family of distributions and is given by

$$
Q(p)=G^{-1}(\vartheta(p))
$$

where

$$
\vartheta(p)=\left(\frac{-\log \left(1-\left(\frac{-\log \left(1-p\left(1-e^{-\lambda}\right)\right)}{\lambda}\right)^{\frac{1}{\beta}}\right)}{\alpha-\log \left(1-\left(\frac{-\log \left(1-p\left(1-e^{-\lambda}\right)\right)}{\lambda}\right)^{\frac{1}{\beta}}\right)}\right)
$$

and $G^{-1}($.$) is the baseline quantile function, thus, the quantile functions of the POGE-$ $\mathrm{HL}$ and POGE-U family of distributions are $Q_{P O G E-H L}(p)=-\log \left(\frac{1-\vartheta(p)}{1+\vartheta(p)}\right)$ and $Q_{P O G E-U}(p)=b \vartheta(p)$, respectively, where $\vartheta(p)$ is given by $(2.13)$.

We now compute the $r^{t h}$ moment, moment generating function of the POGE $(\xi)$ which can be use to study some features and characteristics of the new distribution, such as the mean, variance, skewness and kurtosis etc. For a random variable $X \sim \operatorname{POGE}(\xi)$ then, the $r^{t h}$ moment of $X$ is obtained by

$E\left(X^{r}\right)=\int_{0}^{\infty} x^{r} f(x) d x$

By applying (2.10), we have

$$
E\left(X^{r}\right)=\sum_{i, j=0}^{\infty} \sum_{k, l=0}^{\infty} \varrho_{(i, j, k, l)} \int_{0}^{\infty} x^{r} f^{*}(x ; k+l+1, \zeta) d x,
$$

hence,

$$
E\left(X^{r}\right)=\sum_{i, j=0}^{\infty} \sum_{k, l=0}^{\infty} \varrho_{(i, j, k, l)} E\left(Y_{e}^{r}\right)
$$


where $E\left(Y_{e}^{r}\right)$ is the $r^{t h}$ moment of the exponentiated $G(x ; \zeta)$ distribution with power parameter $k+l+1$. Therefore, we can use (2.14) to compute the $r^{t h}$-moment of the POGE-HL using the $r^{t h}$-moment of the generalized half logistic distribution.

2.1. Lemma. Let $X$ be a random variable that follow generalized half logistic $G H L(\gamma)$ distribution, then, for $r \in \mathbb{N}$, the $r^{\text {th }}$ moment of $X$ is given by

$$
E\left(X^{r}\right)_{G H L}=2 \gamma \sum_{w=0}^{\infty}\left(\begin{array}{c}
-(\gamma+1) \\
w
\end{array}\right)(-1)^{r} B_{0 r}(\gamma, w+1) .
$$

For a random variable $\mathrm{X}$ that follows POGE-HL distribution, the $r^{\text {th }}$ moment of $\mathrm{X}$ can be computed by putting (2.15) in (2.14) when $\gamma=k+l+1$, as

$$
E\left(X^{r}\right)_{P O G E-H L}=\sum_{i, j, k=0}^{\infty} \sum_{l, w=0}^{\infty} \varrho_{(i, j, k, l, w)}^{*} B_{0 r}(k+l+1, w+1),
$$

where

$$
\begin{aligned}
\varrho_{(i, j, k, l, w)}^{*} & =\left(\begin{array}{c}
\beta(i+1)-1 \\
j
\end{array}\right)\left(\begin{array}{c}
-(k+2) \\
l
\end{array}\right)\left(\begin{array}{c}
-(k+l+2) \\
w
\end{array}\right) \\
& \times \frac{2(-1)^{i+j+k+r} \alpha^{k} \lambda^{i+1}(j+1)^{k}}{\left(1-e^{-\lambda}\right) i ! k !} .
\end{aligned}
$$

2.2. Lemma. Let $X$ be a random variable that follow generalized Uniform $G U(0, b)$ distribution, with power parameter $k+l+1$, then, for $r \in \mathbb{N}$, the $r^{\text {th }}$ moment of $X$ is given by

$$
E\left(X^{r}\right)_{G U}=\frac{(k+l+1) b^{r}}{k+l+r+1}
$$

hence, the $r^{t h}$ moment of $X \sim$ POGE-U is obtained by substituting (2.17) in (2.14) as

$$
E\left(X^{r}\right)_{P O G E-U}=\sum_{i, j=0}^{\infty} \sum_{k, l=0}^{\infty} \varrho_{(i, j, k, l)} \frac{(k+l+1) b^{r}}{(k+l+r+1)} .
$$

Figure 2 and 3 provide the plots for the mean and variance of the POGE-HL and POGE-U distributions.
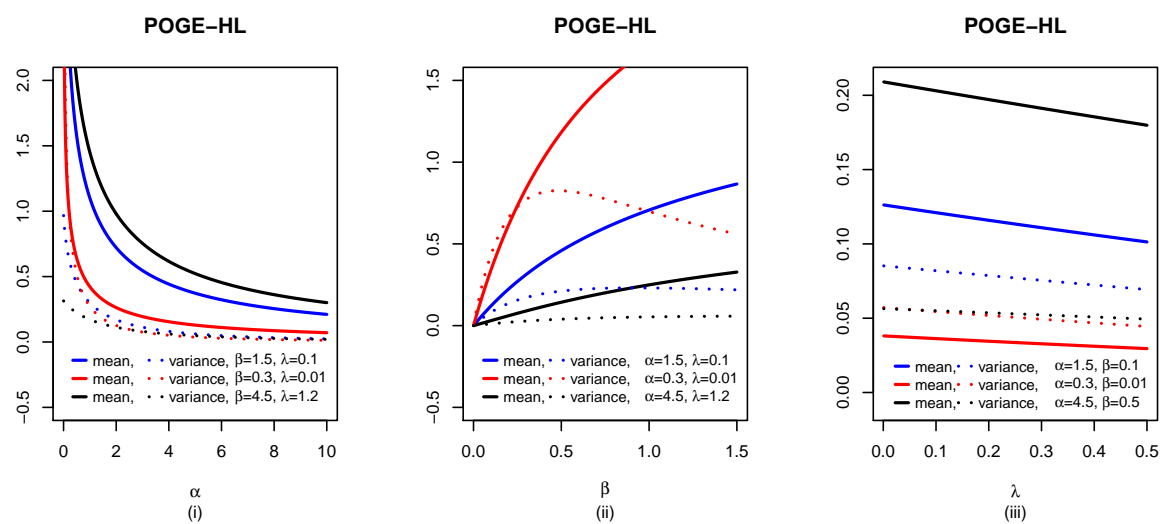

Figure 2. Plots of Mean and Variance: (i) for $\alpha>0$ and some values of $\beta$ and $\lambda$, (ii) for $\beta>0$ and some values of $\alpha$ and $\lambda \&$ (iii) for $\lambda>0$ and some values of $\alpha$ and $\beta$ for the POGE-HL distributions. 

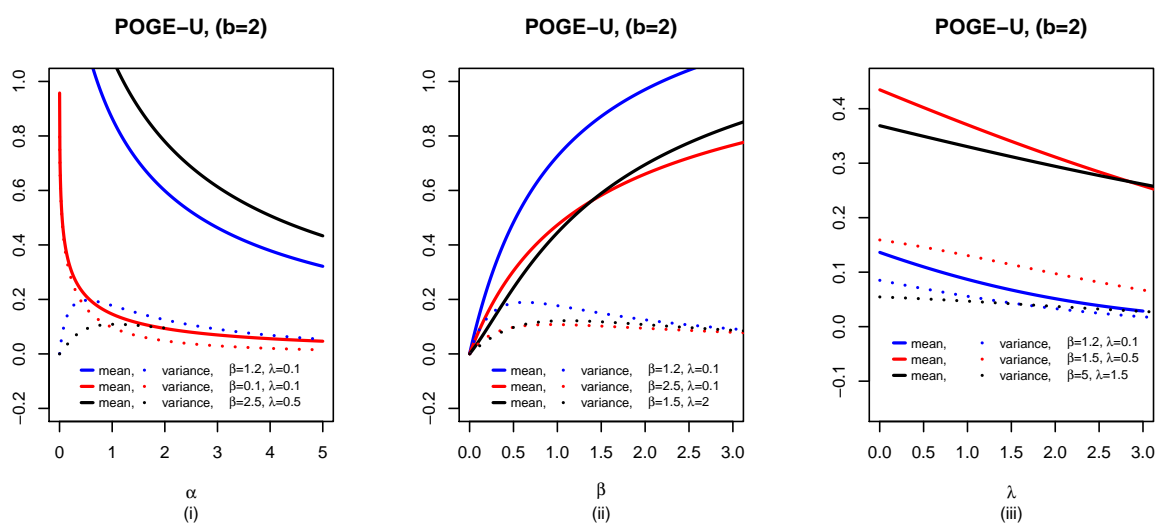

Figure 3. Plots of Mean and Variance: (i) for $\alpha>0$ and some values of $\beta, \lambda$ and $b=2$ (ii) for $\beta>0$ and some values of $\alpha, \lambda$ and $b=2$ $\&$ (iii) for $\lambda>0$ and some values of $\alpha, \beta$ and $b=2$ for the POGE-U distributions.

The moment generating function (mgf) of the POGE distribution can be computed from

$$
M_{X}(t)=\sum_{r=0}^{\infty} \frac{t^{r}}{r !} E\left(X^{r}\right)
$$

Substituting (2.14) in (2.19) gives the mgf of the POGE distribution. Also the mgf of the POGE-HL can be obtained by putting (2.16) in (2.19) as

$$
M_{X}(t)_{P O G E-H L}=\sum_{r, i, j=0}^{\infty} \sum_{k, l, w=0}^{\infty} \tilde{\varrho}_{(i, j, k, l, r, w)} B_{0 r}(k+l+1, w+1),
$$

where

$$
\begin{aligned}
\tilde{\varrho}_{(i, j, k, l, r, w)} & =\left(\begin{array}{c}
\beta(i+1)-1 \\
j
\end{array}\right)\left(\begin{array}{c}
-(k+2) \\
l
\end{array}\right)\left(\begin{array}{c}
-(k+l+2) \\
w
\end{array}\right) \\
& \times \frac{2(-1)^{i+j+k+r} t^{r} \alpha^{k} \lambda^{i+1}(j+1)^{k}}{\left(1-e^{-\lambda}\right) i ! k ! r !} .
\end{aligned}
$$

For the POGE-U, we get the moment generating function by substituting (2.18) in (2.19) as

$$
M_{X}(t)_{P O G E-U}=\sum_{r, i, j=0}^{\infty} \sum_{k, l=0}^{\infty} \varrho_{(i, j, k, l)} \frac{(k+l+1)(b t)^{r}}{(k+l+r+1) r !} .
$$

One of the alternative measures for the skewness and kurtosis of a distribution which can be more appropriate for the POGE distribution are the Bowley skewness (B) and Moores kurtosis (M). These measures are defined as follow

$$
B=\frac{Q_{(3 / 4)}+Q_{(1 / 4)}-2 Q_{(2 / 4)}}{Q_{(3 / 4)}-Q_{(1 / 4)}},
$$




$$
M=\frac{Q_{(3 / 8)}-Q_{(1 / 8)}+Q_{(7 / 8)}-Q_{(5 / 8)}}{Q_{(6 / 8)}-Q_{(2 / 8)}},
$$

respectively. We now demonstrate how the parameters $\alpha$ and $\beta$ effect the behavior of the skewness and kurtosis of the POGE-HL and POGE-U distributions for fixed values of parameters $\lambda$ and $b$. Figure 4 illustrate the plot of Bowley skewness and Moores kurtosis of the POGE-HL distribution while Figure 5 for the POGE-U distribution.
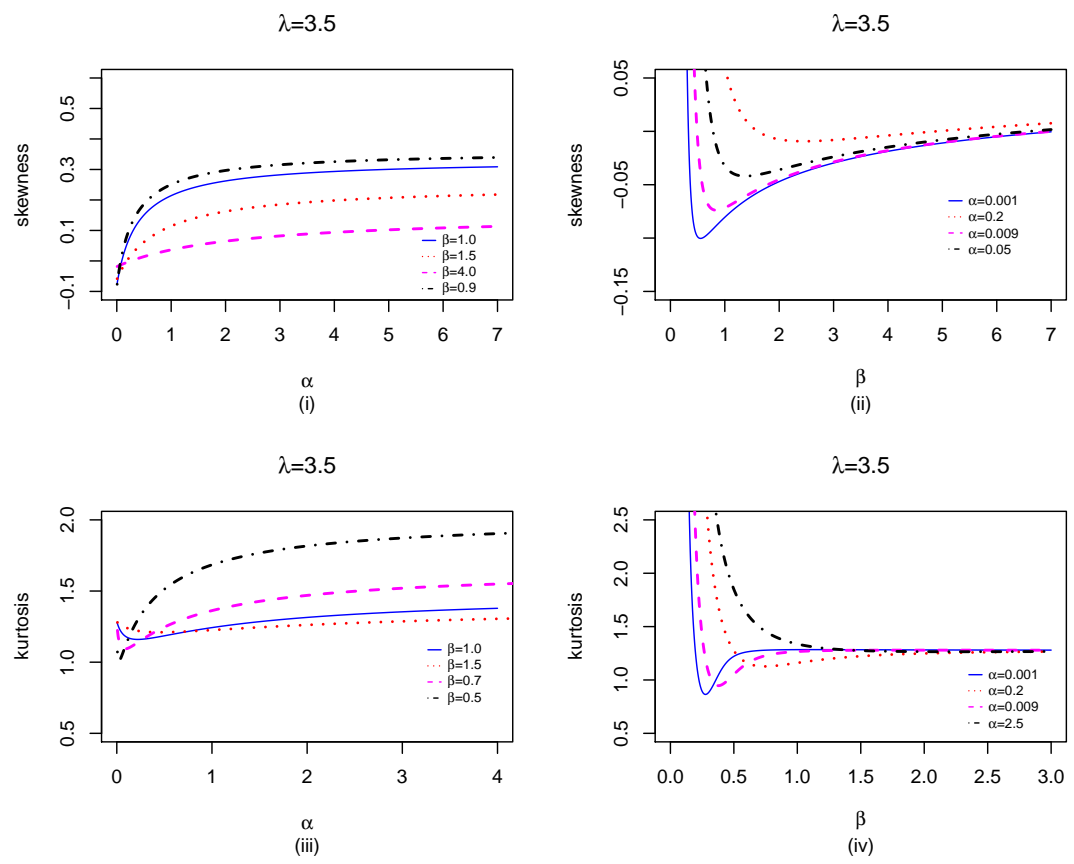

Figure 4. Plots of skewness (i) \& (ii) and kurtosis (iii) \& (iv) of POGE-HL. 


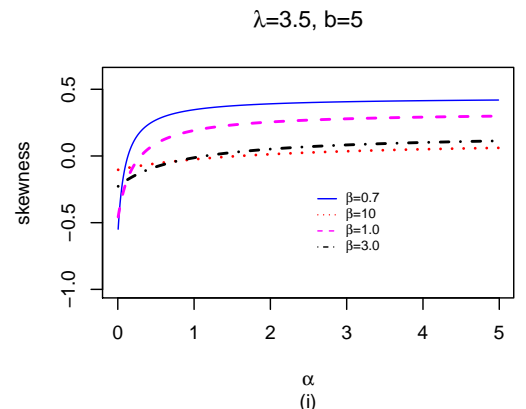

$\lambda=3.5, b=5$

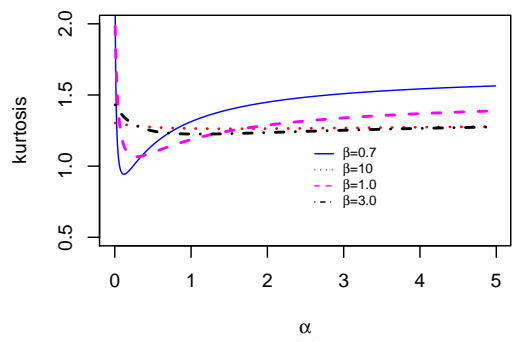

$\alpha$
(iii)

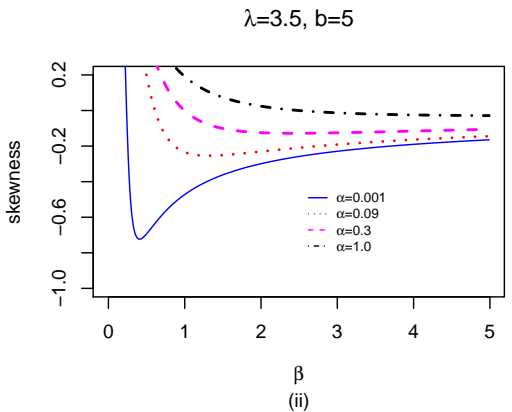

$\lambda=3.5, b=5$

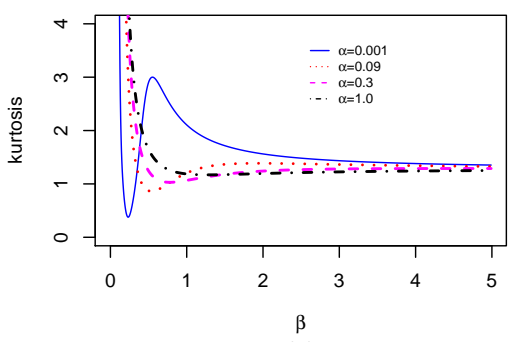

(iv)

Figure 5. Plots of skewness (i) \& (ii) and kurtosis (iii) \& (iv) of POGE-U.

2.4. Order statistics. Order statistics are very important tool in many areas of statistical theory and applications such as in quality control and reliability. Let $X_{1}, X_{2}, \cdots, X_{n}$ be a random sample of size $\mathrm{n}$ obtained from POGE distribution, then, the density $f_{j: n}(x)$ of the $j^{\text {th }}$ order statistic $X_{j: n}, j=1,2,3, \cdots, n$, can be express as

$$
\begin{aligned}
f_{x_{j}: n}(x ; \alpha, \beta, \lambda, \theta) & =\frac{n !}{(j-1) !(n-j) !} f(x)(F(x))^{j-1}(1-F(x))^{n-j}, \\
& =\sum_{i=0}^{n-j} \frac{n !(-1)^{i}}{(j-1) !(n-j-i) ! i !} f(x)(F(x))^{j+i-1} .
\end{aligned}
$$

Substituting $F(x)$ and $f(x)$ given by (2.1) and (2.2) respectively, and using the binomial expansion and some algebraic manipulations we have

$$
f_{j: n}(x ; \xi)=\sum_{i=0}^{n-j} \sum_{k=0}^{i+j-1} \Psi_{i, k} f(x ; \alpha, \beta, \lambda(k+1), \zeta),
$$

where

$$
\Psi_{i, k}=\left(\begin{array}{c}
i+j-1 \\
k
\end{array}\right) \frac{n !(-1)^{i+k}\left(1-e^{-\lambda(k+1)}\right)}{(k+1)\left(1-e^{-\lambda}\right)^{i+j}(j-1) !(n-j-i) ! i !},
$$

and $f(x ; \alpha, \beta, \lambda(k+1), \zeta)$ is the pdf of POGE with parameters $\alpha, \beta, \lambda(k+1)$ and $\zeta$. The $r^{t h}$ moment of the $j^{\text {th }}$ order statistics $X_{j: n}$ is give by 


$$
E\left(X_{j: n}^{r}\right)=\sum_{i=0}^{n-j} \sum_{k=0}^{i+j-1} \Psi_{i, k} \int_{0}^{\infty} x^{r} f(x ; \alpha, \beta, \lambda(k+1), \zeta) d x
$$

or

$$
E\left(X_{j: n}^{r}\right)=\sum_{i=0}^{n-j} \sum_{k=0}^{i+j-1} \Psi_{i, k} E\left(Y_{\delta}^{r}\right)
$$

where $Y_{\delta} \sim \operatorname{POGE}(\alpha, \beta, \lambda(k+1), \zeta)$ and $E\left(Y_{\delta}^{r}\right)$ is the $r^{t h}$ moment of the POGE distribution with parameters $\alpha, \beta, \lambda(k+1)$ and $\zeta$.

2.5. Entropy. Entropy is a measure of uncertainty of a random variable. In this section, we consider the two important entropies known as the Shannon and Renyi entropies. The Shannon entropy measure is defined by $E[-\log f(x)]$. For a random variable $X$ having POGE distribution, the Shannon entropy of $X$ can be computed by considering lemma 2.3 and proposition 2.4 as follows.

2.3. Lemma. For $\rho_{1}, \rho_{2}, \rho_{3}, \in \mathbb{R}$, let

$$
\varphi\left(\rho_{1}, \rho_{2}, \rho_{3}, \xi\right)=\int_{0}^{1} \frac{x^{\rho_{1}} e^{-\alpha\left(\frac{x}{1-x}\right)}\left[1-e^{-\alpha\left(\frac{x}{1-x}\right)}\right]^{\rho_{2}-1}}{(1-x)^{\rho_{3}}} e^{-\lambda\left[1-e^{-\alpha\left(\frac{x}{1-x}\right)}\right]^{\beta}} d x
$$

then,

$$
\varphi\left(\rho_{1}, \rho_{2}, \rho_{3}, \xi\right)=\sum_{i, j=0}^{\infty} \sum_{k, l=o}^{\infty} \varsigma_{i, j, k, l}(\alpha, \lambda, \rho)\left(\begin{array}{c}
\rho_{2}+\beta i-1 \\
j
\end{array}\right)\left(\begin{array}{c}
-\rho_{3}-k \\
l
\end{array}\right)
$$

where $\varsigma_{i, j, k, l}\left(\alpha, \lambda, \rho_{1}\right)=\frac{(-1)^{i+j+k+l} \alpha^{k} \lambda^{i}}{i ! j !\left(\rho_{1}+k+l+1\right)}$.

Proof. By Taylor expansion of $e^{-\lambda\left[1-e^{-\alpha\left(\frac{x}{1-x}\right)}\right]^{\beta}}$, and for $\rho_{2}+\beta i>0$ real and non integer we apply (2.7) then, exponential expansion and generalized binomial expansions.

2.4. Proposition. Let $X$ be a random variable with pdf given by (2.2), then,

$$
\begin{aligned}
E(\log \bar{G}(X)) & =\left.\frac{\alpha \beta \lambda}{\left(1-e^{-\lambda}\right)} \frac{\partial}{\partial t} \varphi(0, \beta, 2-t, \xi)\right|_{t=0}, \\
E\left(\frac{G(X)}{G(X)}\right) & =\frac{\alpha \beta \lambda}{\left(1-e^{-\lambda}\right)} \varphi(1, \beta, 3, \xi), \\
E\left(\log \left(1-e^{-\alpha \frac{G(X)}{G(X)}}\right)\right) & =\left.\frac{\alpha \beta \lambda}{\left(1-e^{-\lambda}\right)} \frac{\partial}{\partial t} \varphi(0, t+\beta, 2, \xi)\right|_{t=0}, \\
E\left(\left(1-e^{-\alpha \frac{G(X)}{G(X)}}\right)^{\beta}\right) & =\frac{\alpha \beta \lambda}{\left(1-e^{-\lambda}\right)} \varphi(0,2 \beta, 2, \xi) .
\end{aligned}
$$

Proof. Considering the transformation $Y=G(x, \zeta), y \in(0,1)$, and Lemma 2.3. 
Thus, the Shannon entropy of POGE distribution is obtained as

$$
\begin{aligned}
E[-\log f(X)] & =-\log \left(\frac{\alpha \beta \lambda}{1-e^{-\lambda}}\right)-E(\log g(X))-2 E(\log \bar{G}(X)) \\
& -\alpha E\left(\frac{G(X)}{G(X)}\right)-(\beta-1) E\left(\log \left(1-e^{-\alpha \frac{G(X)}{G(X)}}\right)\right) \\
& +\lambda E\left(\left(1-e^{-\alpha \frac{G(X)}{G(X)}}\right)^{\beta}\right)
\end{aligned}
$$

and hence,

$$
\begin{aligned}
E[-\log f(X)] & =-\log \left(\frac{\alpha \beta \lambda}{1-e^{-\lambda}}\right)-E(\log g(X)) \\
& -\left.\frac{2 \alpha \beta \lambda}{\left(1-e^{-\lambda}\right)} \frac{\partial}{\partial t} \varphi(0, \beta, 2-t, \xi)\right|_{t=0}+\frac{\alpha^{2} \beta \lambda}{\left(1-e^{-\lambda}\right)} \varphi(1, \beta, 3, \xi) \\
& -\left.\frac{\alpha \beta(\beta-1) \lambda}{\left(1-e^{-\lambda}\right)} \frac{\partial}{\partial t} \varphi(0, t+\beta, 2, \xi)\right|_{t=0}+\frac{\alpha \beta \lambda^{2}}{\left(1-e^{-\lambda}\right)} \varphi(0,2 \beta, 2, \xi) .
\end{aligned}
$$

For a random variable $\mathrm{X}$ with pdf (2.2), the Renyi entropy is defined by $I_{R(\rho)}=(1-\rho)^{-1} \log \left[\int_{0}^{\infty} f(x)^{\rho} d x\right]$, where $\rho>0$ and $\rho \neq 1$. We first simplify

$f^{\rho}(x ; \xi)=\frac{(\alpha \beta \lambda)^{\rho} g^{\rho}(x)}{\bar{G}(x)^{2 \rho}\left(1-e^{-\lambda}\right)^{\rho}} e^{-\alpha \rho \frac{G(x)}{G(x)}}\left(1-e^{-\alpha \frac{G(x)}{G(x)}}\right)^{\rho(\beta-1)} e^{-\lambda \rho\left(1-e^{-\alpha \frac{G(x)}{G(x)}}\right)^{\beta}}$,

expanding $e^{-\lambda \rho\left(1-e^{-\alpha \frac{G(x)}{G(x)}}\right)^{\beta}}$, and then, expanding $\left(1-e^{-\alpha \frac{G(x)}{G(x)}}\right)^{\rho(\beta-1)-\rho}$ using

(2.7) and finally expanding the exponential function obtained, arrive at

$$
\begin{aligned}
f^{\rho}(x ; \xi) & =\jmath_{\alpha, \beta, \lambda, \rho} \sum_{i, j=0}^{\infty} \frac{(-1)^{i+j}(\lambda \rho)^{i}}{i !}\left(\begin{array}{c}
\beta(\rho+i)-\rho \\
j
\end{array}\right) \\
& \times \sum_{k=0}^{\infty} \frac{(-1)^{k}(\rho+j)^{k}}{k !} \frac{g^{\rho}(x) G^{k}(x)}{(1-G)^{k+2 \rho}} .
\end{aligned}
$$

In addition, expanding $(1-G(x))^{-(k+2 \rho)}$ using generalized binomial expansion yields

$$
f^{\rho}(x ; \xi)=\sum_{l=0}^{\infty} J_{i, j, k}(\alpha, \beta, \lambda, \rho) g^{\rho}(x) G^{k+l}(x),
$$

where

$$
\begin{aligned}
J_{i, j, k}(\alpha, \beta, \lambda, \rho) & =\jmath_{\alpha, \beta, \lambda, \rho} \sum_{i, j=0}^{\infty} \frac{(-1)^{i+j}(\lambda \rho)^{i}}{i !}\left(\begin{array}{c}
\beta(\rho+i)-\rho \\
j
\end{array}\right) \\
& \times \sum_{k=0}^{\infty} \frac{(-1)^{k+l}(\rho+j)^{k}}{k !}\left(\begin{array}{c}
-(k+2 \rho) \\
l
\end{array}\right)
\end{aligned}
$$

and $\jmath_{\alpha, \beta, \lambda, \rho}=\left(\frac{\alpha \beta \lambda}{1-e^{-\lambda}}\right)^{\rho}$.

Thus,

$$
I_{R(\rho)}=\frac{1}{1-\rho} \log \left[\sum_{l=0}^{\infty} J_{i, j, k}(\alpha, \beta, \lambda, \rho) \int_{-\infty}^{\infty} g^{\rho}(x) G^{k+l}(x) d x\right]
$$


2.6. The stress-strength parameter. Suppose that, the random variables $X_{1}$ and $X_{2}$ are independent with $\operatorname{POGE}\left(\alpha, \beta_{1}, \lambda_{1}, \zeta\right)$ and $\operatorname{POGE}\left(\alpha, \beta_{2}, \lambda_{2}, \zeta\right)$ distributions, respectively. In reliability studies, the stress-strength model describes the life of a component which has a random strength $X_{1}$ that is subjected to a random stress $X_{2}$. If $X_{1}>X_{2}$ the component will function satisfactorily and when $X_{2}>X_{1}$ the component will fail because the stress applied exceed the strength. The reliability of a component $\mathrm{R}=\mathrm{P}\left(X_{2}<X_{1}\right)=\int_{0}^{\infty} f_{1}(x ; \xi) F_{2}(x ; \xi) d x$, has many applications in different fields of engineering such as maintenance in electric power, electronics and in study of fatigue failure of aircraft structures. The reliability function can be computed as follows.

$$
\begin{aligned}
f_{1}(x ; \xi) F_{2}(x ; \xi) & =\frac{f_{1}(x ; \xi)}{\left(1-e^{-\lambda_{2}}\right)} \\
& -\left(\frac{\alpha \beta_{1} \lambda_{1} g(x) e^{-\alpha \frac{G(x)}{G(x)}}\left(1-e^{-\alpha \frac{G(x)}{G(x)}}\right)^{\beta_{1}-1}}{\bar{G}(x)^{2}\left(1-e^{-\lambda_{1}}\right)\left(1-e^{-\lambda_{2}}\right)}\right. \\
& e^{\left.-\lambda_{1}\left(1-e^{-\alpha \frac{G(x)}{G(x)}}\right)^{\beta_{1}} e^{\left.-\lambda_{2}\left(1-e^{-\alpha \frac{G(x)}{G(x)}}\right)^{\beta_{2}}\right)}\right)} . \\
& e^{-\lambda_{2}\left(1-e^{-\alpha \frac{G(x)}{G(x)}}\right)^{\beta_{2}}} \text { and some algebraic substitutions we have }
\end{aligned}
$$

$$
\begin{aligned}
\int_{0}^{\infty} f_{1}(x ; \xi) F_{2}(x ; \xi) d x & =\frac{1}{\left(1-e^{-\lambda_{2}}\right)} \\
& -\left(\frac{\alpha \beta_{1} \lambda_{1}}{\left(1-e^{-\lambda_{1}}\right)\left(1-e^{-\lambda_{2}}\right)} \sum_{w=0}^{\infty} \frac{(-1)^{w} \lambda_{2}^{w}}{w !}\right. \\
& \int_{0}^{1} \frac{e^{-\alpha\left(\frac{x}{1-x}\right)}}{(1-x)^{2}}\left(1-e^{-\alpha\left(\frac{x}{1-x}\right)}\right)^{\beta_{1}+\beta_{2} w-1} \\
& \left.e^{-\lambda_{1}\left(1-e^{-\alpha\left(\frac{x}{1-x}\right)}\right)^{\beta_{1}}} d x\right) .
\end{aligned}
$$

Considering (2.24), we obtain

$$
\begin{aligned}
R & =\frac{1}{\left(1-e^{-\lambda_{2}}\right)} \\
& -\frac{\alpha \beta_{1} \lambda_{1}}{\left(1-e^{-\lambda_{1}}\right)\left(1-e^{-\lambda_{2}}\right)} \sum_{w=0}^{\infty} \frac{(-1)^{w} \lambda_{2}^{w}}{w !} \varphi^{*}\left(0, \beta_{1}+\beta_{2} w, 2, \xi\right),
\end{aligned}
$$

where

$$
\begin{aligned}
\varphi^{*}\left(0, \beta_{1}+\beta_{2} w, 2, \xi\right) & =\sum_{i, j=0}^{\infty} \sum_{k, l=o}^{\infty} \varsigma_{i, j, k, l}^{*}\left(\alpha, \lambda_{1}\right) \\
& \times\left(\begin{array}{c}
\beta_{2} w+\beta_{1}(i+1)-1 \\
j
\end{array}\right)\left(\begin{array}{c}
-2-k \\
l
\end{array}\right),
\end{aligned}
$$

$\varsigma_{i, j, k, l}^{*}\left(\alpha, \lambda_{1}\right)=\frac{(-1)^{i+j+k+l} \alpha^{k} \lambda_{1}^{i}}{i ! j !(k+l+1)}$ and $\varphi^{*}($.$) follow similar to \varphi($.$) given in Lemma 2.3.$ 


\section{Estimation and inference}

In this section, we estimate the unknown parameters of the POGE distribution by the method of maximum likelihood. Let $X_{i}(i=1,2, \cdots, n)$ be a random sample of size $\mathrm{n}$ obtained from the POGE distribution with observed values $x_{1}, x_{2}, \ldots, x_{n}$. The MLEs of $\Theta=(\alpha, \beta, \lambda, \zeta)^{T}$ are obtained by maximization of the $\log$-likelihood function $(\log \ell(\Theta))$ given by

$$
\begin{aligned}
\log \ell(\Theta) & =n \log \alpha+n \log \beta+n \log \lambda+\sum_{i=1}^{n} \log g\left(x_{i} ; \zeta\right)-2 \sum_{i=1}^{n} \log \bar{G}\left(x_{i} ; \zeta\right) \\
& -n \log \left(1-e^{-\lambda}\right)-\alpha \sum_{i=1}^{n} H\left(x_{i} ; \zeta\right)+(\beta-1) \sum_{i=1}^{n} \log \left(1-e^{-\alpha H\left(x_{i} ; \zeta\right)}\right) \\
& -\lambda \sum_{i=1}^{n}\left(1-e^{-\alpha H\left(x_{i} ; \zeta\right)}\right)^{\beta}
\end{aligned}
$$

The MLEs of $\Theta=(\alpha, \beta, \lambda, \zeta)^{T}$ say $\hat{\Theta}=(\hat{\alpha}, \hat{\beta}, \hat{\lambda}, \hat{\zeta})^{T}$ are obtained simultaneously by solving the $\frac{\partial \ell}{\partial \alpha}=\frac{\partial \ell}{\partial \beta}=\frac{\partial \ell}{\partial \lambda}=\frac{\partial \ell}{\partial \zeta}=0$ numerically.

In the case of POGE-U distribution where $\zeta=b$ the upper boundary of the distribution support is restricted by the choice of the parameter $b$, therefore, for an ordered random sample of size $n$, say $x_{1: n}, x_{2: n}, x_{3: n}, \cdots, x_{n: n}$ which follow POGE-U distribution, we can obtain the estimates of $\alpha, \beta$ and $\lambda$ by the numerical solutions of $\frac{\partial \ell}{\partial \alpha}=\frac{\partial \ell}{\partial \beta}=\frac{\partial \ell}{\partial \lambda}=0$ for a fixed $b>X_{n: n}$. Due to the different choices of $b>X_{n: n}$, the procedure may produces a large bias for the MLEs of $\alpha, \beta$ and $\lambda$. [4] proved using simulation study in the estimation of the parameters of Weibull-pareto (WP) distribution, that, the modified maximum likelihood method (MMLE) may be a better choice for reducing the bias. For the interval estimate and hypothesis tests of the parameters we required $J(\Theta)$ the $4 \times 4$ Fisher information matrix which is given by $J(\Theta)=-\left(\frac{\partial^{2}(\log \ell(\Theta))}{\partial \Theta \partial \Theta^{T}}\right)$. The elements of $J(\Theta)$ can be obtained from the author under request.

3.1. Simulation. Simulations have been carried out in order to investigate the estimators of the parameters of the POGE-HL distribution. We generated 10,000 samples of size $n=100,200,300$ and 400 from the POGE-HL distribution for some values of $\alpha, \beta$ and $\lambda$. The MLEs are determined through solving the nonlinear equations $\frac{\partial \ell}{\partial \alpha}=\frac{\partial \ell}{\partial \beta}=\frac{\partial \ell}{\partial \lambda}=0$, where $H\left(x_{i} ; \zeta\right)=H\left(x_{i}\right)=\left(\frac{e^{x_{i}}-1}{2}\right)$. The MLEs and their standard deviations $(s d)$ are presented in Table 1 below. The results show that each MLE converges to its true value in all cases when the sample size increases and the standard errors of the MLEs decrease as the sample size increases. 
Table 1. MLEs and standard deviations for some selected values of parameters.

\begin{tabular}{|c|c|c|c|c|c|c|c|c|c|}
\hline \multirow{2}{*}{$\begin{array}{r}\text { Sample size } \\
n\end{array}$} & \multicolumn{3}{|c|}{ Selected values } & \multicolumn{3}{|c|}{ Estimated values } & \multicolumn{3}{|c|}{ Standard deviations } \\
\hline & $\alpha$ & $\beta$ & $\lambda$ & $\hat{\alpha}$ & $\hat{\beta}$ & $\hat{\lambda}$ & $s d(\hat{\alpha})$ & $s d(\hat{\beta})$ & $s d(\hat{\lambda})$ \\
\hline \multirow[t]{11}{*}{100} & 0.5 & 0.5 & 0.5 & 0.4986 & 0.5193 & 0.7250 & 0.1243 & 0.0758 & 0.9787 \\
\hline & 1.0 & 1.0 & 1.0 & 1.0075 & 1.0059 & 1.2157 & 0.2910 & 0.1380 & 1.3936 \\
\hline & 1.0 & 0.5 & 1.0 & 1.0351 & 0.5049 & 1.1099 & 0.3131 & 0.0727 & 1.1891 \\
\hline & 2.0 & 1.0 & 2.0 & 2.1947 & 0.9863 & 1.9984 & 0.7992 & 0.1249 & 1.6034 \\
\hline & 1.3 & 2.5 & 1.0 & 1.2759 & 2.4866 & 1.1932 & 0.3118 & 0.3787 & 1.3459 \\
\hline & 0.03 & 0.3 & 0.5 & 0.0309 & 0.3102 & 0.6401 & 0.0091 & 0.0478 & 0.7005 \\
\hline & 1.2 & 1.5 & 2.0 & 1.3322 & 1.4828 & 1.8496 & 0.4345 & 0.1969 & 1.5159 \\
\hline & 0.2 & 0.5 & 0.2 & 1.9424 & 0.5307 & 0.5295 & 0.0437 & 0.0770 & 0.8741 \\
\hline & 0.7 & 0.6 & 0.5 & 0.6932 & 0.6214 & 0.7430 & 0.1711 & 0.0911 & 1.0488 \\
\hline & 4.0 & 5.0 & 2.0 & 4.4856 & 5.2579 & 1.5705 & 1.0749 & 1.0748 & 1.4329 \\
\hline & 0.3 & 0.4 & 3.0 & 0.4100 & 0.3937 & 2.7477 & 0.2351 & 0.0447 & 1.5294 \\
\hline \multirow[t]{11}{*}{200} & 0.5 & 0.5 & 0.5 & 0.4976 & 0.5096 & 0.6143 & 0.0903 & 0.0573 & 0.7108 \\
\hline & 1.0 & 1.0 & 1.0 & 0.9935 & 0.9978 & 1.1659 & 0.2302 & 0.1032 & 1.2135 \\
\hline & 1.0 & 0.5 & 1.0 & 1.0150 & 0.5015 & 1.0543 & 0.2290 & 0.0559 & 0.8789 \\
\hline & 2.0 & 1.0 & 2.0 & 2.0804 & 0.9862 & 2.1215 & 0.6723 & 0.0884 & 1.4900 \\
\hline & 1.3 & 2.5 & 1.0 & 1.2795 & 2.4664 & 1.1990 & 0.2754 & 0.2689 & 1.2607 \\
\hline & 0.03 & 0.3 & 0.5 & 0.0306 & 0.3038 & 0.5591 & 0.0072 & 0.0367 & 0.5207 \\
\hline & 1.2 & 1.5 & 2.0 & 1.2589 & 1.4780 & 2.0325 & 0.3755 & 0.1381 & 1.4370 \\
\hline & 0.2 & 0.5 & 0.2 & 0.1960 & 0.5189 & 0.3957 & 0.0306 & 0.0568 & 0.5527 \\
\hline & 0.7 & 0.6 & 0.5 & 0.6931 & 0.6111 & 0.6321 & 0.1252 & 0.0685 & 0.7534 \\
\hline & 4.0 & 5.0 & 2.0 & 4.1441 & 5.0277 & 2.0423 & 1.0281 & 0.8107 & 1.6150 \\
\hline & 0.3 & 0.4 & 3.0 & 0.3542 & 0.3964 & 2.9379 & 0.1583 & 0.0316 & 1.2982 \\
\hline \multirow[t]{11}{*}{300} & 0.5 & 0.5 & 0.5 & 0.5003 & 0.5064 & 0.5602 & 0.0736 & 0.0488 & 0.5052 \\
\hline & 1.0 & 1.0 & 1.0 & 1.0002 & 0.9955 & 1.0867 & 0.1939 & 0.0867 & 0.9918 \\
\hline & 1.0 & 0.5 & 1.0 & 1.0155 & 0.5006 & 1.0164 & 0.1874 & 0.0462 & 0.6735 \\
\hline & 2.0 & 1.0 & 2.0 & 2.0403 & 0.9879 & 2.1430 & 0.5873 & 0.0723 & 1.3808 \\
\hline & 1.3 & 2.5 & 1.0 & 1.2783 & 2.4691 & 1.1893 & 0.2524 & 0.2239 & 1.1753 \\
\hline & 0.03 & 0.3 & 0.5 & 0.0302 & 0.3009 & 0.5252 & 0.0054 & 0.0341 & 0.4474 \\
\hline & 1.2 & 1.5 & 2.0 & 1.2272 & 1.4783 & 2.0887 & 0.3313 & 0.1114 & 1.3169 \\
\hline & 0.2 & 0.5 & 0.2 & 0.1966 & 0.5140 & 0.3448 & 0.0254 & 0.0463 & 0.4528 \\
\hline & 0.7 & 0.6 & 0.5 & 0.6958 & 0.6058 & 0.5734 & 0.1020 & 0.0572 & 0.5837 \\
\hline & 4.0 & 5.0 & 2.0 & 4.0995 & 4.9897 & 2.0346 & 0.9173 & 0.6676 & 1.4149 \\
\hline & 0.3 & 0.4 & 3.0 & 0.3352 & 0.3978 & 3.0026 & 0.1306 & 0.0260 & 1.2458 \\
\hline \multirow[t]{11}{*}{400} & 0.5 & 0.5 & 0.5 & 0.4994 & 0.5043 & 0.5467 & 0.0647 & 0.0426 & 0.4420 \\
\hline & 1.0 & 1.0 & 1.0 & 1.0009 & 0.9965 & 1.0607 & 0.1692 & 0.0773 & 0.8407 \\
\hline & 1.0 & 0.5 & 1.0 & 1.0116 & 0.4994 & 1.0036 & 0.1603 & 0.0408 & 0.5315 \\
\hline & 2.0 & 1.0 & 2.0 & 2.0109 & 0.9909 & 2.1480 & 0.5247 & 0.0628 & 1.2195 \\
\hline & 1.3 & 2.5 & 1.0 & 1.2850 & 2.4671 & 1.1314 & 0.2278 & 0.1934 & 1.0662 \\
\hline & 0.03 & 0.3 & 0.5 & 0.0301 & 0.3029 & 0.5378 & 0.0042 & 0.0248 & 0.3733 \\
\hline & 1.2 & 1.5 & 2.0 & 1.2144 & 1.4810 & 2.1164 & 0.3074 & 0.0974 & 1.2441 \\
\hline & 0.2 & 0.5 & 0.2 & 0.1965 & 0.5114 & 0.3183 & 0.0221 & 0.0415 & 0.3813 \\
\hline & 0.7 & 0.6 & 0.5 & 0.6989 & 0.6041 & 0.5441 & 0.0886 & 0.0507 & 0.4702 \\
\hline & 4.0 & 5.0 & 2.0 & 4.0629 & 4.9759 & 2.0647 & 0.8604 & 0.6116 & 1.3101 \\
\hline & 0.3 & 0.4 & 3.0 & 0.3260 & 0.3979 & 3.0115 & 0.1127 & 0.0223 & 1.0614 \\
\hline
\end{tabular}




\section{Application}

In this section, we provide applications of the POGE distribution to two real data set in order to illustrate the importance of the POGE family of distributions. For comparison, we fitted the first data set with the following distributions, the POGE-HL, POE-HL, odd generalized exponential half logistic (OGE-HL), Poisson half logistic (PHL) by [2], exponentiated half logistic (EHL) by [15], Olapade-generalized half logistic (OLGHL) by [32], generalized exponential (GE) by [14], generalized exponential Poisson (GEP) by [8], BurrXII-Poisson (BXIIP) by [38] and generalized BurrXII-Poisson (GBXIIP) by [28]. For the second data we fitted the POGE-U distribution and compare the fit with the POE-U, odd generalized exponential uniform (OGE-U), gamma-uniform (GU) by [41], generalized modified weibull (GMW) by [11], beta modified weibull (BMW) by [36], beta weibull (BW) by [18], modified weibull distribution (MW) by [17], generalized linear failure rate (GLFR) by [35], generalized linear exponential (GLE) by [20], exponentiated generalized linear exponential (EGLE) by [34] and some family of the generalized modified weibull-power series (GMWPS) such as generalized modified weibull-Poisson (GMWP), generalized modified weibull-Geometric (GMWG) and generalized modified weibull-Logarithmic (GMWL) distributions proposed by [5]. The MLEs of the parameters for each model are computed and the three criteria for model selection are used for comparison namely the Akaike information criterion (AIC), consistent Akaike information criterion (CAIC) and Bayesian information criterion, also the goodness-of-fit statistics known as the Kolmogorov Smirnov (KS) is used to compare the fit of the new POGE family and other competing models. The model with the least values of AIC, CAIC, BIC and KS fit the data better than the other models. The MLEs of each model parameters $(\hat{\Theta})$ and numerical values of these measures for the first data set are given in Table 2 while for the second data set in Table 3 below. The first data set is given by [31] the data set are the 100 observations on breaking stress of carbon fibers (in Gba): $0.39,0.81,0.85,0.98,1.08,1.12,1.17,1.18,1.22,1.25,1.36,1.41,1.47,1.57,1.57,1.59$, $1.59,1.61,1.61,1.69,1.69,1.71,1.73,1.80,1.84,1.84,1.87,1.89,1.92,2.00,2.03,2.03$, $2.05,2.12,2.17,2.17,2.17,2.35,2.38,2.41,2.43,2.48,2.48,2.50,2.53,2.55,2.55,2.56$, $2.59,2.67,2.73,2.74,2.76,2.77,2.79,2.81,2.81,2.82,2.83,2.85,2.87,2.88,2.93,2.95$, $2.96,2.97,2.97,3.09,3.11,3.11,3.15,3.15,3.19,3.19,3.22,3.22,3.27,3.28,3.31,3.31$, $3.33,3.39,3.39,3.51,3.56,3.60,3.65,3.68,3.68,3.68,3.70,3.75,4.20,4.38,4.42,4.70$, $4.90,4.91,5.08,5.56$.

The second data set is the lifetimes of fifty devices provided by [1], the data set are: $.1, .2,1,1,1,1,1,2,3,6,7,11,12,18,18,18,18,18,21,32,36,40,45,46,47,50,55$, $60,63,63,67,67,67,67,72,75,79,82,82,83,84,84,84,85,85,85,85,85,86,86$.

As you can see form Table 2 that, the POGE-HL distribution represent the first data sets better than the other models, while Table 3 shows that, the POGE-U distribution provide better fit than the other distributions. Figure 6 illustrate the histogram and cdfs for the first data set and the fitted POGE-HL distribution, while Figure 7 give the quantile-quantile plot and profile-log likelihood functions of the fitted POGE-HL distribution for the first data set. Figure 8 shows the histogram and cdfs for the second data set and the fitted POGE-U distribution, and Figure 9 provides the quantile-quantile plot and profile-log likelihood functions of the fitted POGE-U distribution for the second data set. 
Table 2. MLEs, $\ell(\Theta)$, AIC, CAIC, BIC, KS and P-value for the first data.

\begin{tabular}{lcccccccccc}
\hline Model & $\alpha$ & $\beta$ & $\lambda$ & $a$ & $\ell(\Theta)$ & AIC & CAIC & BIC & KS & P-value \\
\hline First data set & & & & & & & & & & \\
POGE-HL & 0.0385 & 1.1977 & 4.0520 & - & -143.75 & 293.49 & 287.56 & 301.31 & 0.0513 & 0.955 \\
POE-HL & 0.0272 & - & 4.3643 & - & -145.12 & 294.23 & 290.27 & 299.44 & 0.0695 & 0.720 \\
OGE-HL & 0.0809 & 0.9181 & - & - & -152.57 & 311.13 & 305.20 & 318.95 & 0.1238 & 0.093 \\
PHL & 0.0451 & - & -16.9271 & - & -196.60 & 397.20 & 393.24 & 402.41 & 0.3195 & $2.7 \mathrm{e}-9$ \\
EHL & 1.0796 & 4.9029 & - & - & -144.75 & 293.50 & 289.54 & 298.71 & 0.0981 & 0.291 \\
OLGHL & 0.9713 & 0.5000 & - & - & -181.17 & 366.34 & 362.38 & 371.55 & 0.2721 & $7.4 \mathrm{e}-7$ \\
GE & 1.0132 & 7.7882 & - & - & -146.18 & 296.37 & 292.41 & 301.58 & 0.1077 & 0.196 \\
GEP & 1.0131 & 7.7901 & 0.0008 & - & -146.18 & 298.37 & 292.43 & 306.18 & 0.1074 & 0.199 \\
BXIIP & 5.9395 & 0.1874 & $3.4 e-6$ & - & -189.48 & 384.97 & 379.03 & 392.78 & 0.2714 & $8.0 \mathrm{e}-7$ \\
GBXIIP & 1.3502 & 2.1236 & $1.1 e-4$ & 13.7368 & -158.77 & 325.54 & 317.62 & 335.96 & 0.1380 & 0.044 \\
\hline
\end{tabular}

Table 3. MLEs, $\ell(\Theta)$, AIC, CAIC, BIC, KS and P-value for the second data.

\begin{tabular}{lccccccccccccc}
\hline Model & $\alpha$ & $\beta$ & $\lambda$ & $a$ & $b$ & $\gamma$ & $\theta$ & $\ell(\Theta)$ & AIC & CAIC & BIC & KS & P-value \\
\hline Second data set & & & & & & & & & & & & & \\
POGE-U & 0.022 & 0.371 & 1.769 & - & 87.010 & - & - & -206.68 & 419.34 & 413.47 & 425.08 & 0.0923 & 0.754 \\
POE-U & 0.293 & - & 1.638 & - & 97.100 & - & - & -229.55 & 463.10 & 459.18 & 466.92 & 0.2346 & 0.007 \\
OGE-U & 0.034 & 0.282 & - & - & 87.001 & - & - & -207.97 & 421.93 & 416.06 & 427.67 & 0.1471 & 0.208 \\
GU & 0.267 & 51.942 & - & 0.09 & 86.713 & - & - & -207.33 & 418.65 & 414.83 & 426.30 & 0.1520 & 0.198 \\
EGLE & - & - & - & $3.3 e-3$ & $1.7 \mathrm{e}-4$ & 4.564 & 0.112 & -224.34 & 456.67 & 448.85 & 464.32 & 0.1475 & 0.206 \\
GLE & - & - & - & $9.6 e-3$ & $4.5 \mathrm{e}-4$ & 0.730 & - & -235.93 & 477.85 & 471.98 & 483.59 & 0.1598 & 0.139 \\
GLFR & - & - & - & $3.8 e-3$ & $3.1 \mathrm{e}-4$ & - & 0.533 & -233.15 & 472.29 & 466.42 & 478.03 & 0.1620 & 0.129 \\
GMWP & $5.4 e-8$ & 0.134 & 0.084 & - & - & 2.137 & 0.638 & -220.88 & 451.75 & 441.98 & 461.31 & 0.1400 & 0.281 \\
GMWL & 2.130 & 2.682 & 0.013 & - & - & 0.278 & 1.000 & -217.77 & 445.53 & 435.76 & 455.09 & 0.1311 & 0.357 \\
GMWG & $9.4 e-8$ & 0.123 & 0.075 & - & - & 2.228 & 0.464 & -220.78 & 451.55 & 441.78 & 461.11 & 0.1346 & 0.326 \\
GMW & $1.0 e-5$ & 0.065 & - & 0.223 & - & 1.371 & - & -221.40 & 452.81 & 442.99 & 460.46 & 0.1464 & 0.234 \\
BMW & $2.4 e-4$ & 0.054 & - & 0.197 & 0.165 & 1.380 & - & -220.28 & 450.56 & 440.78 & 460.12 & 0.1303 & 0.364 \\
BW & $1.0 e-5$ & - & - & 0.129 & 0.070 & 3.320 & - & -223.11 & 454.22 & 446.40 & 461.87 & 0.1246 & 0.419 \\
MW & 0.062 & 0.023 & - & - & - & 0.355 & - & -226.16 & 460.31 & 452.44 & 466.05 & 0.1338 & 0.332 \\
\hline
\end{tabular}
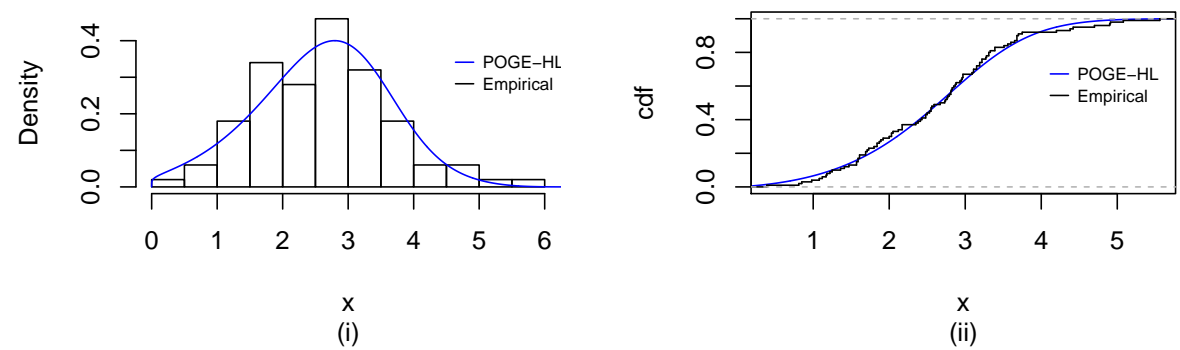

Figure 6. Fitted pdf (i) and cdf (ii) of the POGE-HL for the first data set. 

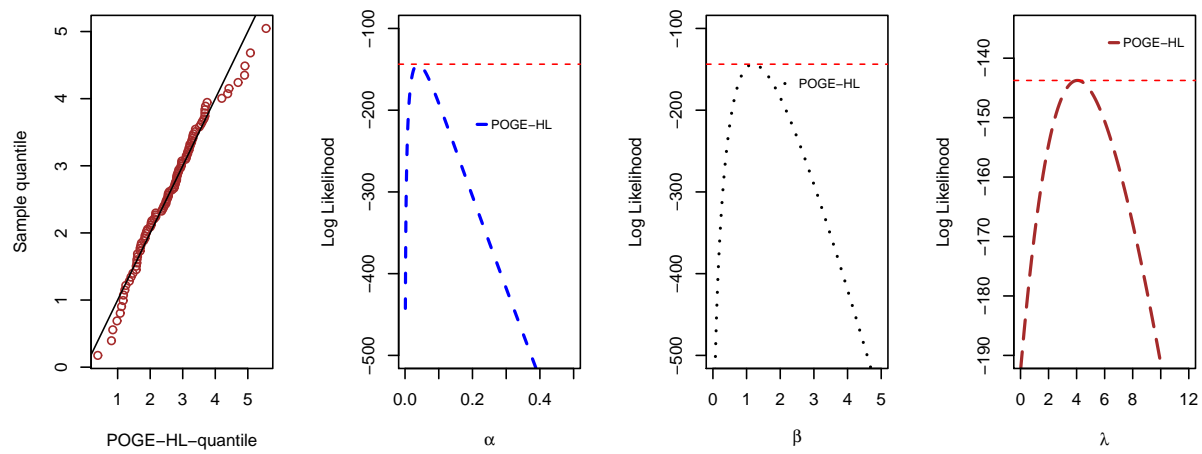

Figure 7. Quantile-quantile Plot and Profile log-likelihood function of the POGE-HL for the first data set.
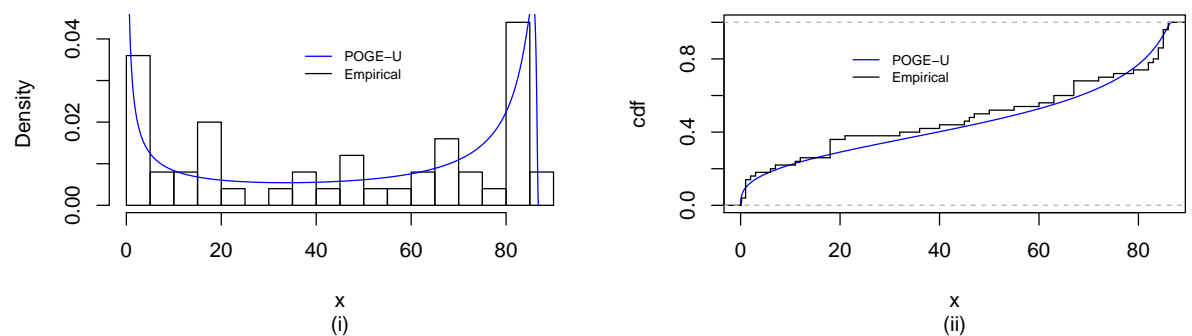

Figure 8. Fitted pdf (i) and cdf (ii) of the POGE-U for the second data set.
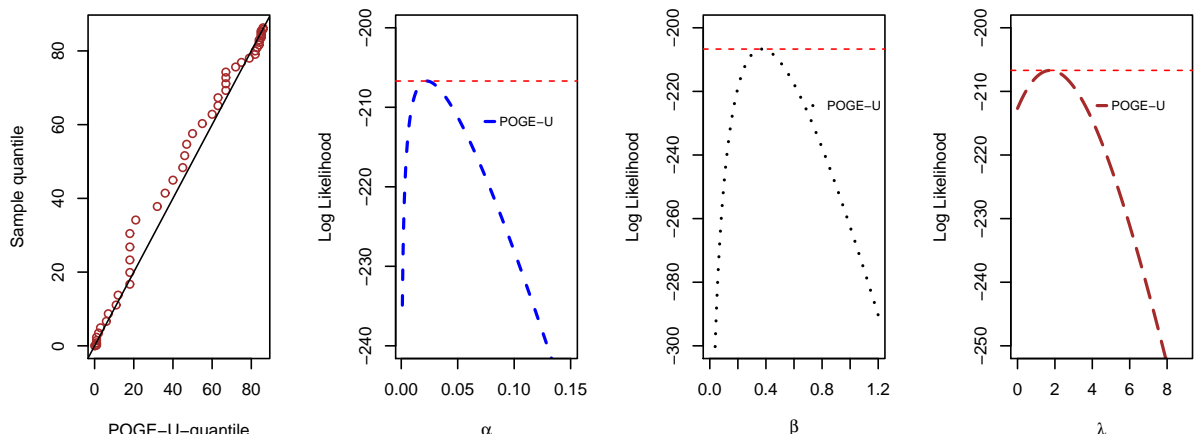

Figure 9. Quantile-quantile Plot and Profile log-likelihood function of the POGE-U for the second data set

\section{Conclusions}

We have introduced a new family of lifetime distributions called the Poisson odd generalized exponential distribution (POGE). We discussed two special cases of the POGE family of distributions, namely the Poisson odd generalized exponential-half logistic (POGE-HL) and the Poisson odd generalized exponential-uniform (POGE-U) family of 
distributions. Several mathematical and statistical properties of this model are derived and studied, such as infinite series of the pdf, an explicit algebraic expression of its moments, order statistics, Shannon entropy, Renyi entropy and reliability. We discuss the maximum likelihood estimations of the model parameters. Simulation studies are performed for various sample sizes from the POGE-HL distribution in order to assess the performance of proposed maximum likelihood method. Two real applications are used to show the usefulness of the new POGE family of distributions. The result shows that the POGE families (i.e POGE-HL and POGE-U) give a better fit to the two data sets than the other competing models.

Acknowledgements The authors would like to thank the editor and referees for their valuable comments and suggestions which greatly improve the quality of the paper.

\section{References}

[1] Aarset, M. How to identify bathtub hazard rate, IEEE Trans. Reliab 36, 106-108, 1987.

[2] Abdel-Hamid, A. H. Properties, estimations and predictions for a poisson-half logistic distribution based on progressively type-ii censored samples., Applied Mathematical Modelling 40 (15), 7164-7181, 2016.

[3] Adamidis, K. and S. Loukas. A lifetime distribution with decreasing failure rate, Statistics \& Probability Letters 39 (1), 35-42, 1998.

[4] Alzaatreh, A., F. Famoye, and C. Lee. Weibull-pareto distribution and its applications, Communications in Statistics - Theory and Methods 42 (9), 1673-1691, 2003.

[5] Bagheri, S., E. B. Samani, and M. Ganjali. The generalized modified weibull power series distribution: Theory and applications, Computational Statistics \& Data Analysis 94, 136$160,2016$.

[6] Bakouch, H. S., M. A. Jazi, S. Nadarajah, A. Dolati, and R. Roozegar. A lifetime model with increasing failure rate, Applied Mathematical Modelling 38 (32), 5392-5406, 2014.

[7] Bakouch, H. S., M. M. Ristic, A. Asgharzadeh, L. Esmaily, and B. M. Al-Zahrani. An exponentiated exponential binomial distribution with application, Statistics \& Probability Letters 82 (6), 1067-1081, 2012.

[8] Barreto-Souza, W. and F. Cribari-Neto. A generalization of the exponential-poisson distribution, Statistics \& Probability Letters 79 (24), 2493-2500, 2009.

[9] Bourguignon, M., R. B. Silva, and G. M. Cordeiro. The weibull-G family of probability distributions, Journal of Data Science 12 (1), 53-68, 2014.

[10] Cancho, V. G., F. Louzada-Neto, and G. D. Barriga. The poisson-exponential lifetime distribution, Computational Statistics \& Data Analysis 55 (1), 677-68, 2011.

[11] Carrasco, J. M., E. M. Ortega, and G. M. Cordeiro. A generalized modfied weibull distribution for lifetime modeling, Computational Statistics \& Data Analysis 53 (2), 450-462, 2008.

[12] Gomez, Y. M., H. Bolfarine, and H. W. Gomez. A new extension of the exponential distribution, Revista Colombiana de Estadstica 37 (1), 25-34, 2014.

[13] Gui, W., S. Zhang, and X. Lu. The lindley-poisson distribution in lifetime analysis and its properties, Hacettepe Journal of Mathematics and Statistics 43 (6), 1063-1077, 2014.

[14] Gupta, R. D. and D. Kundu. Theory 83 methods: Generalized exponential distributions, Australian \& New Zealand Journal of Statistics 41 (2), 173-188, 1999.

[15] Kantam, R., V. Ramakrishna, and M. Ravikumar. Estimation and testing in type I generalized half logistic distribution, Journal of Modern Applied Statistical Methods 12 (1), 22, 2013.

[16] Kus, C. A new lifetime distribution, Computational Statistics \& Data Analysis 51 (9), 4497-4509, 2007.

[17] Lai, C., M. Xie, and D. Murthy. A modfied weibull distribution, IEEE Transactions on reliability $\mathbf{5 2}$ (1), 33-37, 2003.

[18] Lee, C., F. Famoye, and O. Olumolade. Beta-weibull distribution: some properties and applications to censored data, Journal of modern applied statistical methods 6 (1), 17, 2007. 
[19] Louzada, F., V. Marchi, and J. Carpenter. The complementary exponentiated exponential geometric lifetime distribution, Journal of Probability and Statistics, 2013.

[20] Mahmoud, M. and F. M. A. Alam. The generalized linear exponential distribution, Statistics \& probability letters 80 (11), 1005-1014, 2010.

[21] Mahmoudi, E. and A. A. Jafari. Generalized exponential-power series distributions,Computational Statistics \& Data Analysis 56 (12), 4047-4066, 2012.

[22] Mahmoudi, E. and A. A. Jafari. The compound class of linear failure rate-power series distributions: Model, properties and applications., arXiv preprint arXiv:1402.5282.

[23] Mahmoudi, E. and A. Sepahdar. Exponentiated weibull-poisson distribution: Model, properties and applications, Mathematics and Computers in Simulation 92 76-97, 2013.

[24] Mahmoudi, E., A. Sepahdar, and A. Lemonte. Exponentiated Weibull-logarithmic Distribution: Model, Properties and Applications, ArXiv e-prints. 2014.

[25] Mahmoudi, E. and M. Shiran. Exponentiated weibull-geometric distribution and its applications, arXiv preprint arXiv:1206.4008, 2012a.

[26] Mahmoudi, E. and M. Shiran. Exponentiated weibull power series distributions and its applications, arXiv preprint arXiv:1212.5613, 2012b.

[27] Morais, A. L. and W. Barreto-Souza. A compound class of weibull and power series distributions, Computational Statistics \& Data Analysis 55 (3), 1410-1425, 2011.

[28] Muhammad, M. A generalization of the burrxii-poisson distribution and its applications., Journal of Statistics Applications \& Probability 5 (1), 29-41, 2016.

[29] Nadarajah, S. and F. Haghighi. An extension of the exponential distribution, Statistics 45 (6), 543-558, 2011.

[30] Nadarajah, S. and R. Roozegar. Bivariate weibull-power series class of distributions, Hacettepe Journal of Mathematics and Statistics, 46 (6), 1175-1186, 2017.

[31] Nichols, M. D. and W. Padgett. A bootstrap control chart for weibull percentiles, Quality and reliability engineering international 22 (2), 141-151, 2006.

[32] Olapade, A. The type I generalized half logistic distribution, Journal of Iranian Statistical Society 13 (1), 69-82, 2014.

[33] Ristic, M. M. and S. Nadarajah. A new lifetime distribution, Journal of Statistical Computation and Simulation 84 (1), 135-150, 2014.

[34] Sarhan, A. M., E.-B. A. Abd, and I. A. Alasbahi. Exponentiated generalized linear exponential distribution, Applied Mathematical Modelling 37 (5), 2838-2849, 2013.

[35] Sarhan, A. M. and D. Kundu. Generalized linear failure rate distribution, Communications in StatisticsTheory and Methods 38 (5), 642-660, 2009.

[36] Silva, G. O., E. M. Ortega, and G. M. Cordeiro. The beta modfied weibull distribution, Lifetime Data Analysis 16 (3), 409-430, 2010.

[37] Silva, R. B., M. Bourguignon, C. R. Dias, and G. M. Cordeiro. The compound class of extended weibull power series distributions, Computational Statistics \& Data Analysis 58, 352-367, 2013.

[38] Silva, R. B., G. M. Cordeiro, et al. The burr xii power series distributions: A new compounding family, Brazilian Journal of Probability and Statistics 29 (3), 565-589, 2015.

[39] Tahir, M. H., G. M. Cordeiro, M. Alizadeh, M. Mansoor, M. Zubair, and G. G. Hamedani. The odd generalized exponential family of distributions with applications, Journal of Statistical Distributions and Applications 2 (1), 1-28, 2015.

[40] Tahmasebi, S. and A. A. Jafari. Generalized gompertz-power series distributions, arXiv preprint arXiv:1508.07634, 2015 .

[41] Torabi, H. and N. M. Hedesh. The gamma-uniform distribution and its applications, Kybernetika 48 (1), 16-30, 2012. 